\title{
Business intelligence en plataforma libre, basado en indicadores de gestión, para coordinaciones zonales
}

\author{
Business intelligence in free platform, based on management indicators, for \\ coordination's zone
}

Fabián Patricio Londo Yachambay. ${ }^{1}$, Narcisa de Jesús Sánchez Salcán. ${ }^{2}$, \& Oscar Omar Espindola Lara. ${ }^{3}$

Recibido: 14-07-2019 / Revisado: 23-07-2019 /Aceptado: 15-08-2019/ Publicado: 10-09-2019

\begin{abstract}
.
DOI: https://doi.org/10.33262/cienciadigital.v3i3.4.868 The Geographic Information Systems (GIS) and its application in various planning topics such as the transport of belongings and products, GIS have essentially focused on the mobility of people in the urban environment. At present, the problem for government entities is: to promote the movement of citizens, optimize routes, improve frequency times and guarantee the quality of public transport. The research is based on the theoretical principles of transport geography, explores concepts of accessibility and spatial mobility to indicate trends in residential activity, such as the center and some peripheral areas of the city of Riobamba. The investigative process was based on the discovery of data, starting with the extraction of information, then the collection and classification of geographic files, data tables and information provided by entities of the sectional government, which were processed and transformed to be represented in maps of: population density, transport routes and educational infrastructure density.

Results were obtained that allowed to identify the imbalance in both the spatial distribution, density of transport routes and the insufficient integration of the service. Bus lines mostly converge to the city center, with low levels of response times depriving users of decentralized and inclusive mobility. To associate the nodes and arcs connected to the transport network, the Graph Theory was used and, based on the morphological characteristics of the city, network analysis was applied for the design of the new transport routes, interconnecting the new nodes and centralities, integrating the new routes, guaranteeing users adequate mobility to public and private services, located in the city of Riobamba.
\end{abstract}

Keywords: Mobility, urban transport, transport network

\footnotetext{
${ }^{1}$ Escuela Superior Politécnica de Chimborazo. Facultad de Administración de Empresas. flondo@espoch.edu.ec

${ }^{2}$ Universidad Nacional de Chimborazo. Facultad de Ciencias de la Educación Humanas y Tecnologías nsanchez@unach.edu.ec

3 Universidad Nacional de Chimborazo. Facultad de Ciencias de la Educación Humanas y Tecnologías oespindola@unach.edu.ec
} 


\section{Resumen}

La nueva estructuración de zonas, distritos y circuitos que lleva adelante el estado ecuatoriano ha generado una centralización de información hacia las nueve zonas que tiene el país, las cuales se están fortaleciendo y consolidando en sus diferentes ámbitos. Sin embargo, es notoria la falta de aplicativos que ayuden a analizar y procesar información para tomar decisiones oportunas y adecuadas en el momento indicado. El desarrollo de un BI (Inteligencia de Negocios) usando software libre como estipula la política de gobierno, permite realizar el procesamiento y análisis de la información de manera rápida, fiable y oportuna mediante la utilización de técnicas estadísticas, para ello se cuenta con una base de indicadores que permitirán tomar decisiones en la parte administrativa y gerencial, el sistema de BI (Inteligencia de Negocios) está dirigido a los Directores de procesos de las Coordinaciones Zonales quienes son los encargados de tomar decisiones para que las políticas a implementar sea de calidad y llegue a todos los ecuatorianos. El Producto final del trabajo son reportes realizados en Pentaho que permiten visualizar estadísticas de salud, extraídos a través de una concentración, procesamiento y análisis de información de todas las unidades de planificación del país.

Palabras claves: Business intelligence, PostgreSQL, Data Warehouse, Pentaho

\section{Introducción.}

En la actualidad la información se ha convertido en un factor imprescindible para el desarrollo de péquelas, medianas y grandes empresas esto sucede en el sector público y privado. Sin embargo, es notoria la falta de aplicativos que ayuden a analizar y procesar información para tomar decisiones oportunas y adecuadas en el momento indicado.

Concentrar una gran cantidad de información no significa que esta lista para ser procesada, esta información debe ser depurada adecuadamente para que, en el momento que se requiera de algún indicador resumido, este sea el más representativo posible.

El desarrollo de un Business Intelligent (Inteligencia de Negocios) utilizando software libre permitirá realizar un almacenamiento, procesamiento y análisis de la información, además que se podrá hacer consultas utilizando estadísticas exploratorias. (Curto, 2010)

Un BI ayudará a obtener información de primera mano es decir datos de diferentes fuentes y distintos programas concentrados en un solo lugar, y que al momento de requerirla para hacer un análisis de información o requerimiento de indicadores permita actuar y procesar datos ante las necesidades existentes, evitando causar pérdidas de tiempo y recursos al momento de solicitar indicadores de para la toma de decisiones. (Manuel P. G., 2104)

\section{Metodología}

Se limitó a conocer causas por las cuales era necesaria la construcción de un BI, se conoce que la información generada por sus diferentes programas de atención y concentrados en diferentes archivos fuente no se lo hace en una sola base, lo que dificulta al momento de requerir información de diferentes programas, en ciertos casos no se tenía definida una base de indicadores que permitan tomar decisiones, ante estas necesidades se hace imprescindible la utilización de un BI el cual será alimentado con información extraída de diferentes fuentes. A la información extraída se realizó un análisis estadístico descriptivo con el propósito de tener una 
panorámica clara sobre la información extraída, por la cantidad de información que se tiene se determinó una muestra para realizar el análisis.

Se conoce que dentro de las políticas del actual del Gobierno Ecuatoriano, es la implementación y utilización de software libre en todas las instituciones y empresas del sector público, por esta razón es necesario la utilización de un software que sea potencialmente eficiente y tenga las características de libre como es el caso de Pentaho que posee dentro de las características más importantes un potente motor para realizar la extracción, trasformación y carga de archivos (ETL) y que abarca en una sola plataforma todas las soluciones para la construcción de un BI, características que son semejantes a la aplicabilidad en él empresas con gran volumen de generación de datos. (Unesco, 2002)

La metodología que se utilizó en el desarrollo del aplicativo es la de Hefestos por las características que encierran bases grandes de datos, las cuales se ajustan a las necesidades de nuestra investigación.

\section{Resultados}

Para consolidar una base de datos se realizó la recolección y consolidación de datos de las 300 unidades operativas de Salud, el proceso se realizó mediante la participación de los 19 distritos que pertenecen a la Coordinación Zonal.

La validación de datos se realizó a través de dos filtros jerárquicos, la revisión en zona a cien unidades operativas, por lo cual existe un $92 \%$ de confiabilidad de la información con un margen de error del $8 \%$.

\section{Ingreso a la consola de Pentaho}

Posterior a la instalación de Pentaho, ingresan a la consola a través de la dirección localhost/8080/ Pentaho por medio del navegador. En la imagen se puede visualizar la acción.

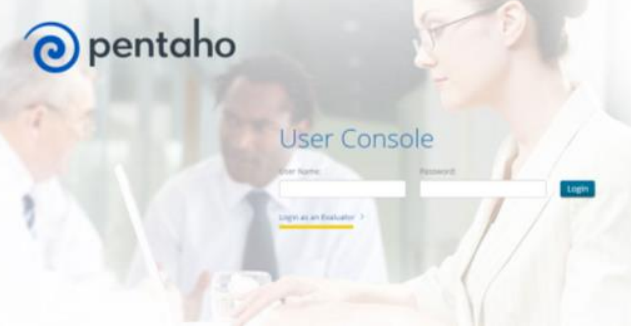

Figura 1. Página de acceso Pentaho

Posteriormente interactúa otra ventana en donde se hace clic en Create New, que les permite escoger con que módulo de Pentaho desean trabajar. El gráfico visualiza lo comentado.

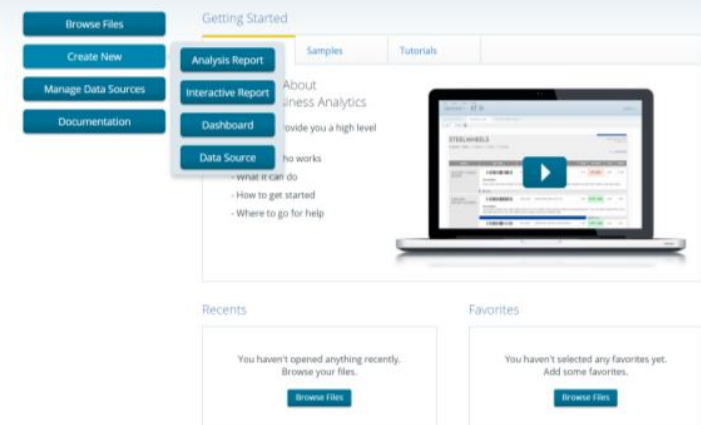

Figura 2. Página de módulos Pentaho 


\section{Creación de reportes}

Para la generación de reportes se ingresa al módulo de Analysis Report, al ingresar a este módulo se visualiza otra pantalla en donde se ingresa el tipo de conexión que se va a trabajar, a continuación, se escogerán las métricas (medidas) que serán útiles para la generación de tablas de información que serán parte de la generación de reportes. En la siguiente figura se ilustra la pantalla donde se realiza este proceso.

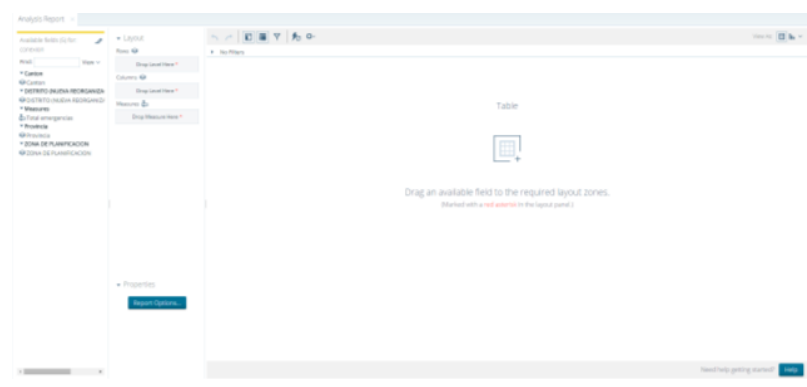

Figura 3. Página de reportes Pentaho

En la imagen que está a continuación, se determina dimensiones y medidas para la generación de cubos de información. El siguiente gráfico muestra un ejemplo.

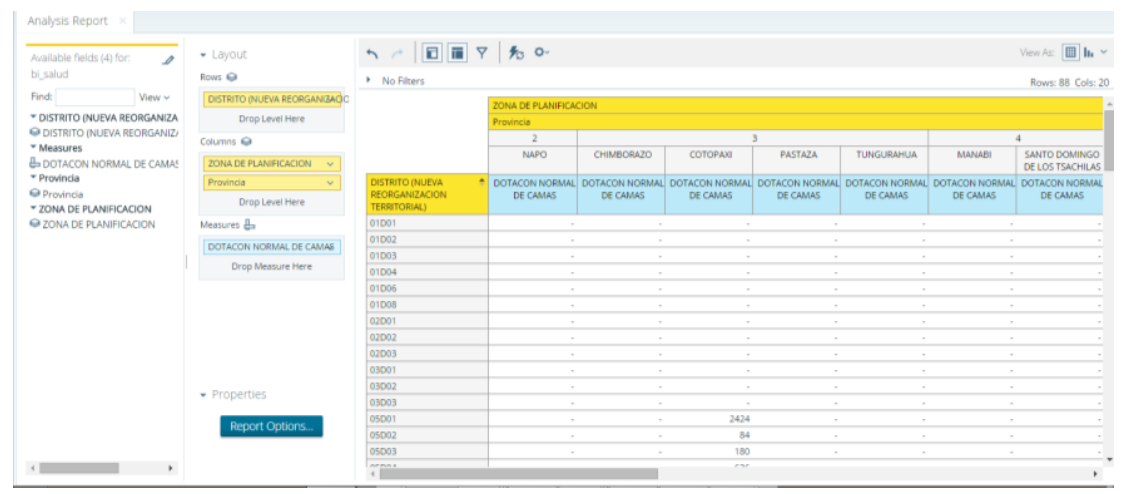

Figura 4. Página de ejemplo Pentaho

\section{Generación de tablas y gráficos}

Para la generación de tablas y gráficos, previamente se debe escoger las dimensiones y medidas que van a dar lugar a la obtención de consultas realizadas. Para realizar una tabla se ubica en la columna de campos que se encuentra en la parte izquierda y se determinan las variables a ser calculadas, luego se arrastran las variables escogidas hacia donde se encuentran las pestañas Rows (filas) y Columns (columnas). En Measure (medida) se define la variable a ser estimada o sobre la cual se va a trabajar todas las consultas, la tabla se forma automáticamente al haber identificado y trasladado variables y medidas a la ventana de dialogo. El gráfico se forma al realizar un clic en la imagen que se encuentra en la parte superior de la consola de trabajo, en donde se permite escoger el tipo de gráfico según las características de la variable a representar. El gráfico que está a continuación muestra la generación de resultados. 


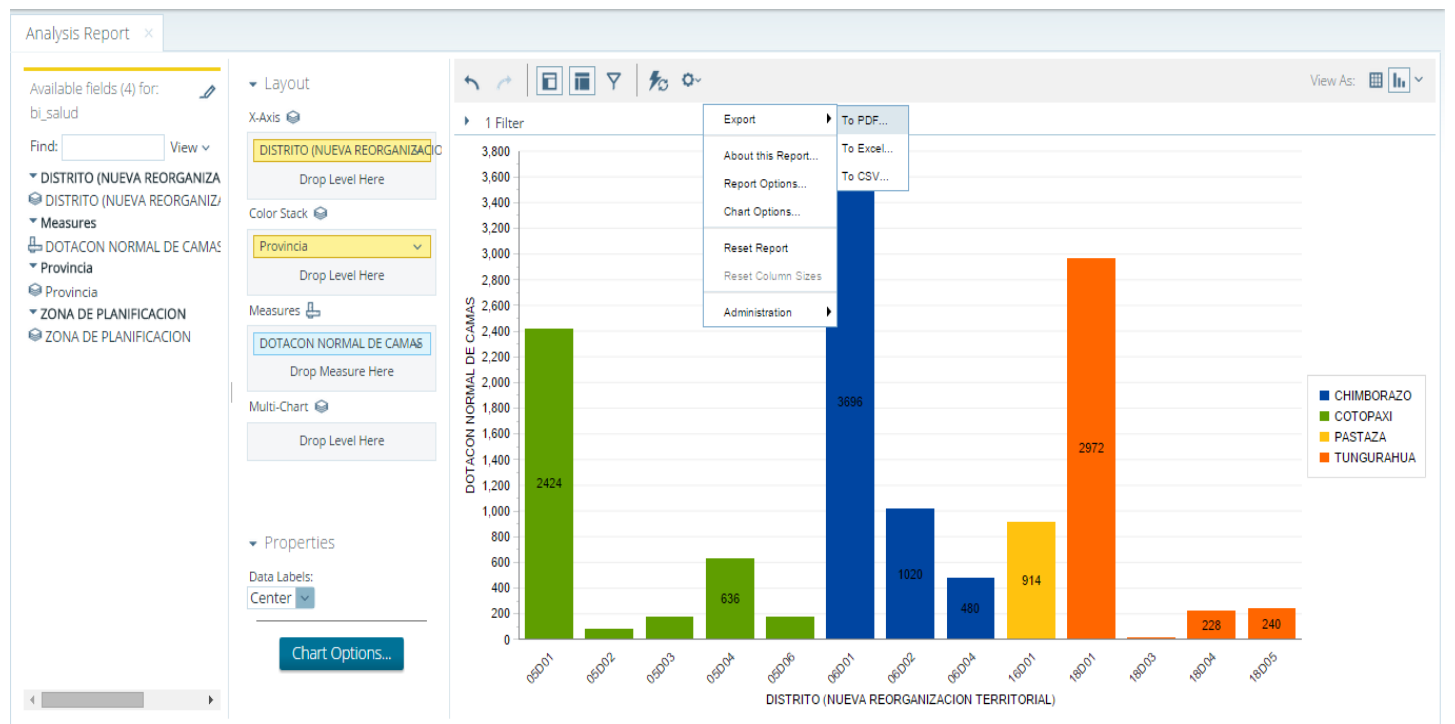

Figura 5. Ejemplo de gráficos Pentaho

\section{Resultados de indicadores}

Para dar lugar a la generación de indicadores propuestos como: nivel ocupación, dotación de camas, total de partos, total de altas, se realiza mediante la construcción de cubos de información para cada indicador, es decir primero se establece las métricas (zona, distrito y provincia), luego se determina las dimensiones que van a generar las tablas y gráficos, de esta forma se obtienen los siguientes resultados.

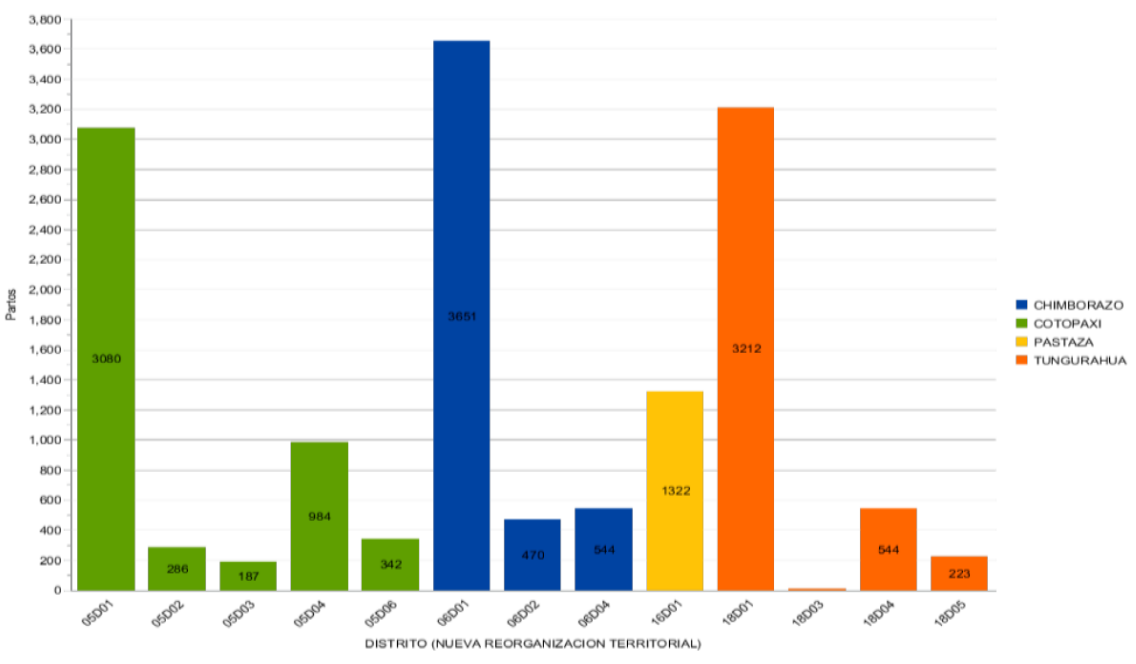

Figura 6. Total partos de la Coordinación Zonal 3 de Salud

En el gráfico se puede observar el total de partos en las 4 provincias de la Coordinación Zonal 3 de Salud, es decir que en la Coordinación Zonal de Salud existió 14859 partos y que la mayor cantidad de partos sucedió en la provincia de Cotopaxi con 4879 partos, lo cual les permite definir si el servicio de Ginecología y Obstetricia que tiene el hospital General de Latacunga cuenta con el número adecuado de camas para atender los partos o es necesario incrementarlas 


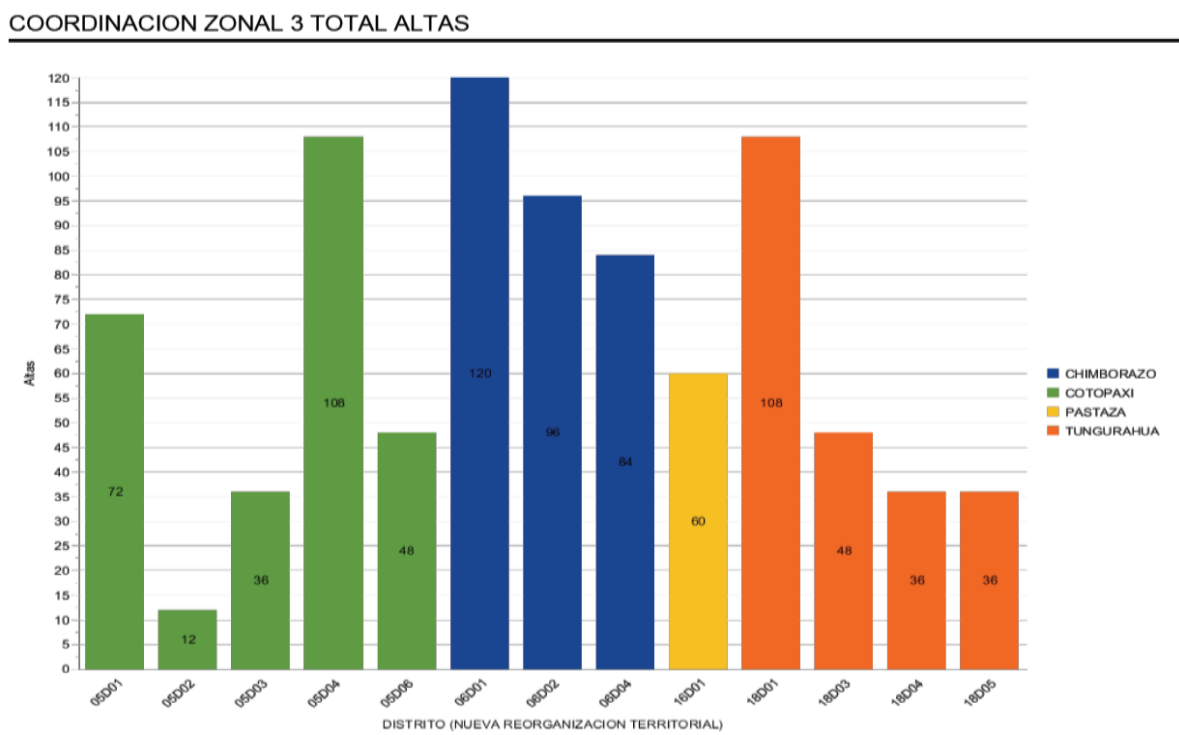

Figura 7. Total de altas de la Coordinación Zonal 3 de Salud

Analizando el total de altas que corresponden a un mes, se observa que el total de altas es de 864 en toda la Coordinación Zonal, existiendo menos altas en la Provincia de Pastaza con 60 altas y en la Provincia de Chimborazo existe el mayor número de altas que son 300, esto les permite considerar si el número de altas realizadas es consecuente con la cantidad de insumos utilizados y con el número de médicos que tiene el hospital.
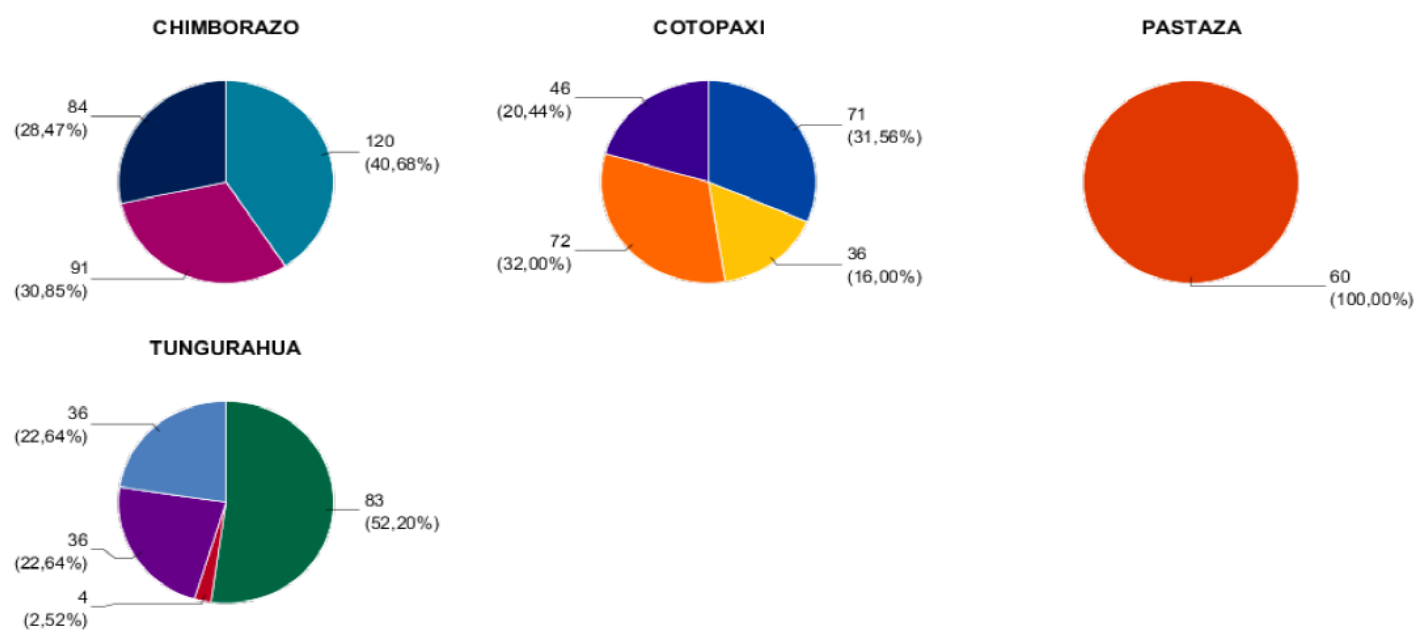

Figura 8. Gráficos comparativos de insumos consumidos en la Coordinación Zonal 3 de Salud

Las cifras nos indican que el porcentaje de ocupación a nivel general es bajo, pero se puede observar que los hospitales Generales son los que tienen el más alto nivel de ocupación, en tanto que los hospitales básicos tienen un bajo nivel de ocupación. Este indicador les permite considerar, si el personal médico con el que cuenta una unidad hospitalaria es el adecuado para satisfacer la demanda de los usuarios que acuden a esta unidad hospitalaria. 


\section{Discusión}

Esta investigación se relaciona y ayuda a tener conceptos definidos sobre la construcción de un Data Warehouse (almacén de Datos) que es una parte que se realiza previo a la construcción del Business Intelligent.

En la Escuela Superior Politécnica de Chimborazo (ESPOCH) existe una tesis cuyo tema es "Estudio de la tecnología Business Intelligence y su aplicación en un modelo de sistema de información gerencial en Petro producción." realizado por Erika Paola Merino Logroño y Hugo Giovanny Vera Flores. Su principal conclusión es que todas las empresas en su mayoría poseen procesos automatizados y sistemas que ayudan en el día a día a sus empleados, por lo cual se puede aseverar que BI es una de las mejores soluciones para empresas del sector público y privado, en cuanto a integración de datos, análisis, consultas y explotación de los mismos. Esta investigación se relaciona en definiciones y metodologías a seguir para la construcción de un BI, además que realiza fundamentaciones para la consolidación de información, que es muy escaso en instituciones públicas y es el propósito de esta investigación.

La integración, entre soluciones tecnológicas, recurso humano y procesos de negocio en un sistema integrado e interrelacionado ha permitido tomar decisiones a partir de la administración de la información, la investigación aporta conocimientos técnicos sobre el diseño de un BI y la administración de instituciones públicas de salud.

La aplicación de un BI en las instituciones públicas de salud de Colombia (IPS) ha permitido tener una cultura organizacional de información, donde la generación de las mismas permite obtener ventajas competitivas para un mejor servicio de salud. [16]

Los modelos estadísticos de clasificación se emplean cotidianamente en salud como sistemas de evaluación que permiten construir grupos homogéneos en la ayuda de pronósticos, diagnósticos, elección de terapias y cuantas situaciones requieran la discretización como herramienta para la toma de decisiones más acertadas. Su utilidad no solo se evidencia en el ámbito médico, sino que muchos de ellos ayudan a mejorar actuaciones y políticas en el sector de la salud pública. Como parte de las tecnologías sanitarias, requieren de una evaluación continua en los diferentes escenarios donde se ejecuten. Por otra parte, emplearlos de manera mecánica, sin una mentalidad crítica, puede traer más riesgos que beneficios, por lo que su poder predictivo o explicativo no justifica que sus resultados se empleen de manera incuestionada. Esta investigación realizada en Colombia para el sector de la Salud Pública de ese país, se relaciona directamente con esta, pues permite considerar parámetros o indicadores que ayudan de gran manera a la toma de decisiones y evaluaciones, que servirá de gran medida en la nueva estructura funcional de salud que tiene Ecuador.

En Cuba se realizó una investigación que consiste en concebir y diseñar una solución matemático computacional para la contextualización de la estrategia sano lógica en diferentes escenarios con vistas a la promoción de salud en términos de la incorporación paulatina de herramientas inteligentes, así como implementar un prototipo como una primera aproximación que permita almacenar, consultar y realizar análisis sobre los datos individuales obtenidos a partir de la aplicación del enfoque senológico con vistas a la toma de decisiones consensuadas. En el análisis del problema a abordar y su contexto ha resultado crucial el intercambio abierto entre los miembros del equipo sano lógico, especialmente, entre los desarrolladores y los profesionales de la salud. El interés informacional se centra en la determinación de las áreas de sana acción a abordar preferentemente en la ruta de salud de una persona. Otras interrogantes que requieren un mayor grado de elaboración corresponden a discernir las áreas de sana acción más frecuentemente detectadas en un consultorio en el último mes; encontrar patrones de 
correlación entre el comportamiento de los miembros de una familia, extrayendo similitudes y diferencias; reconocer las tendencias en la promoción de salud en un territorio.

Durante la etapa de planificación se constató la necesidad de aplicar la estrategia sano lógica como punto de partida para contar con datos almacenados que faciliten no solo la interrelación directa del profesional de la salud y la persona en función de la ruta de salud individual sino también que favorezcan la obtención de información para el accionar colectivo e, incluso, que en el transcurso del tiempo posibiliten investigaciones en comunidades o territorios que propicien decisiones de amplio alcance. (German, 2103)

En Cuba se utilizó información individual para la construcción de un BI que ayudó a obtener indicadores sana lógicos, esta investigación se relaciona porque sigue el propósito de obtener indicadores para tomar decisiones y entender que metodología seguir en la construcción de un BI.

Un estudio realizado en Europa por Information Builders Iberic mostró el costo que tiene la falta de sistemas de toma de decisiones en las organizaciones, según estos datos, el empleado europeo medio pierde una media de 67 minutos diariamente buscando información de la compañía, lo que equivale a un $15,9 \%$ de su jornada laboral. Para una organización de 1.000 empleados que gane unos 50.000 euros al día esto equivale a 7,95 millones de euros al año de salario perdido, todo ello por la búsqueda de información para tomar una decisión. (Lucina, 2013)

El estudio realizado en Europa sirve para dar estadísticas del tiempo y recursos que se pierden al momento de buscar información no centralizada, lo que permite enrumbarse en la construcción de un BI para sectores sociales. Esta investigación se relaciona con la planteada, porque permite ser claros en la utilidad que va resultar su implementación en el sector público, ya que el principal enfoque es tomar decisiones importantes para el sector salud. (Manuel, 2105) Conclusiones.

Se consolidó información del primero, segundo y tercer nivel de atención en Salud, y se analizó utilizando estadística descriptiva (Medidas de Tendencia central y frecuencias) para observar la consistencia de la información, de lo cual se concluye que existe fiabilidad en la información en un $94 \%$.

La utilización de Software Libre para la construcción del BI permitió utilizar las diferentes librerías que dispone Pentaho para la elaboración de un BI y adecuarlas a la información que se tiene en un Coordinación Zonal de Salud, enmarcándonos dentro de las Políticas de Gobierno como es la de "Establecer como política pública para las entidades de la Administración Pública Central la utilización de Software Libre en sus sistemas y equipamientos informáticos". (Subia, 2013)

La concentración de información en diferentes departamentos que la componen y en diferentes formatos, Pentaho por su flexibilidad permitió consolidar la información en una sola base.

De la base de indicadores obtenidos podemos mencionar que existe consistencia en los valores resultantes por la pertinencia que existe en cada uno de los indicadores y su consistencia en la base analizada.

Se concluye que Pentaho a través de sus diversas herramientas permitió realizar el análisis adecuado de la información, obteniendo indicadores de salud fiables que sirvió para tener un enfoque global del servicio que se está brindando. 


\section{Referencias bibliográficas}

Curto, D. (2010). Introducción a Business Intelligent.

German, G. (2103). Estudios de Factibilidad de una Inteligencia de Salud en Hospitales. Costa Rica.

Ilg, P. (2013). Stimuli-responsive hydrogels cross-linked by magnetic nanoparticles. Soft Matter, 9, 3465-3468.

Lucina, G. (2013). Solucion Matematico Computacional para la contextualizacion de una estrategia Sanologica en Cuba. Cuba.

Manuel, P. G. (2104). Definiciòn y Manipulaciòn de Datos.

Manuel, V. (2105). Muestreo Estadístico y Aplicaciones.

Ruiz Estrada, G. (2004). Desarrollo de un Sistema de liberación de fármacos basado en nanopartículas magnéticas recubiertas con Polietilénglicol para el tratamiento de diferentes enfermedades. Madrid: Universidad Autónoma de Madrid. Departamento de Física Aplicada.

Subia, J. (2013). Implementacion de una solucion Bussines Intelligent para el analisis de Gestion del Ministerio de Desarrollo Urbano y Vivienda. Quito.

Unesco. (2002). Guia practica sobre software libre: su selecciòn y aplicacion local en America Latina y el Caribe.

Ilbay Elsi. (2009) Propuesta Metodológica para aplicar Bussines Intelligent caso practico "COHERVIC S.A."

Lemus Juan David, (2005). Aplicación de un Bussines Intelligent en Institucions publicas.

Bouman Rolan. (Penthao Solutions), 2009

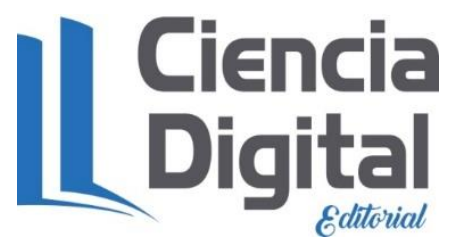




\section{PARA CITAR EL ARTÍCULO INDEXADO.}

Londo Yachambay, F., Sánchez Salcán, N., \& Espindola Lara, O. (2019). Business intelligence en plataforma libre, basado en indicadores de gestión, para coordinaciones zonales. Ciencia Digital, 3(3.4.), 239-248. https://doi.org/10.33262/cienciadigital.v3i3.4.868

El artículo que se publica es de exclusiva responsabilidad de los autores y no necesariamente reflejan el pensamiento de la Revista Ciencia Digital.

El artículo queda en propiedad de la revista y, por tanto, su publicación parcial y/o total en otro medio tiene que ser autorizado por el director de la Revista Ciencia Digital.
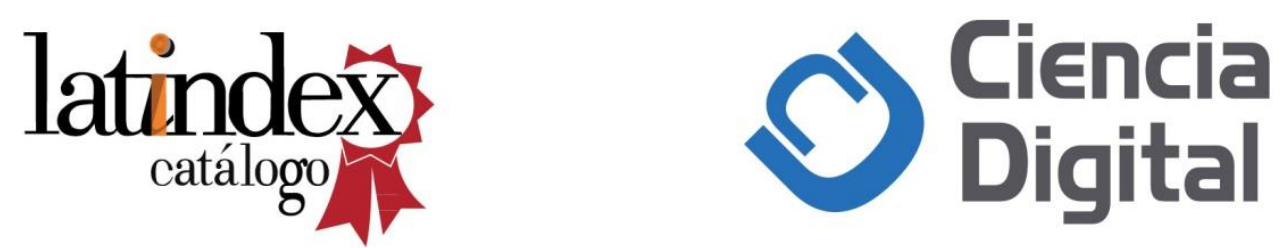